\title{
Barrett's esophagus: lessons from recent clinical trials
}

\author{
Daniela Golger, Andreas Probst, Helmut Messmann
}

Klinikum Augsburg, Germany

Abstract

Data from recent studies cast doubt on former recommendations on diagnosis and management of Barrett's esophagus. Based on latest research findings several Gastroenterological Associations actualized their guidelines and international experts compiled consensus statements as practical help for clinicians. In this review we discuss recent trials and their impact on clinical practice, current recommendations and persisting controversies in Barrett's esophagus.

Keywords Barrett's esophagus, esophageal adenocarcinoma, endoscopic eradication, endoscopic mucosal resection, endoscopic submucosal dissection

Ann Gastroenterol 2016; 29 (4): 1-7

\section{Introduction}

Barrett's esophagus (BE) and its underlying condition, gastroesophageal reflux disease (GERD), predispose to esophageal adenocarcinoma (EAC), a tumor whose incidence has risen dramatically in Western countries during the past decades (in the United States more than 6-fold in 40 years from 0.4 cases per 100000 in 1975 to 2.6 cases per 100000 in 2009 [1]). The prognosis of advanced tumor is poor with a 5 -year survival for distant staged disease of only $2.8 \%$ [1]. If early carcinoma is detected the patient may be offered a potentially curative endoscopic resection (ER), or, if dysplasia is detected, endoscopic ablation to prevent progression to cancer. Hence, screening and surveillance for BE seem rational. Several studies showed that endoscopic surveillance leads to carcinoma detection at earlier stages and to more favorable survival [2]. However, recent studies also showed that the incidence of cancer and the risk of malignant progression among patients with non-dysplastic BE is considerably lower than previously thought [3-5]. Low-grade dysplasia (LGD) on the other hand seems to be an overdiagnosed but underestimated entity [6]. In the past years, tremendous advances evolved as well in ER and ablation techniques as in endoscopic imaging. But is there enough evidence to change practice and what are the lessons learned from recent studies to reconsider diagnostic and therapeutic strategies?

Department of Gastroenterology, Klinikum Augsburg, Germany

Conflict of Interest: None

Correspondence to: Prof. Dr. Helmut Messmann, Department of Gastroenterology, Klinikum Augsburg, Stenglinstr. 2, 86009 Augsburg, Germany.

e-mail: helmut.messmann@klinikum-augsburg.de

Received 21 March 2016; accepted 17 May 2016; published online 14 July 2016

DOI: http://dx.doi.org/10.20524/aog.2016.0070

\section{Epidemiology and cancer risk: should we perform screening?}

Endoscopic screening is a controversial issue. The primary goal of screening is to identify patients with $\mathrm{BE}$ who will benefit from surveillance or therapy to prevent EAC. But first of all who actually should be screened? Known risk factors for BE and EAC are GERD, male sex, white race, older age, obesity, metabolic syndrome, tobacco use, hiatal hernia and a family history of GERD, BE or EAC [7]. The American Gastroenterological Association (AGA) recommends screening for $\mathrm{BE}$ in individuals older than 50 years with symptomatic GERD and at least 1 additional risk factor for EAC [8]. There is no definitive study that supports the assumed benefit of this strategy. But the major dilemma is that a significant proportion of patients with BE and EAC lack reflux symptoms. Approximately 50\% of patients with shortsegment BE deny GERD symptoms and $40 \%$ of patients with EAC reported no history of prior GERD $[9,10]$. Also there are different opinions about the clinical importance of short BE. Another consideration that diminishes the usefulness of screening is the very low risk of malignant progression in nondysplastic BE. Recent population based studies and large metaanalysis showed an annual cancer incidence of only $0.1-0.3 \%$ in these patients and the risk even seems to further decrease over time with follow-up endoscopies showing no progression to dysplasia [3-5,11]. All in all, it is currently difficult to clearly identify the population at risk and more accurate methods for risk stratification are needed. Molecular biomarkers and nonendoscopic technologies for cell collection may help us in the future [12-14]. Promising results have been obtained with the Cytosponge, a cell collection device composed of reticulated foam compressed within a gelatin capsule attached to a string. The capsule is swallowed by the patient and, after $5 \mathrm{~min}$, allowing the dissolution of the gelatin and expansion of the foam, the sponge is retrieved by the operator. During the passage of the sponge cells are absorbed for immunohistochemical analysis. 
In a feasibility study the Cytosponge detected $\mathrm{BE}>1 \mathrm{~cm}$ with $73 \%$ and $\mathrm{BE}>2 \mathrm{~cm}$ with $90 \%$ sensitivity and a specificity of $>90 \%$ [15]. The results of a large multicenter study (BEST2) will provide further information on the diagnostic accuracy. A simulation model, screening a hypothetical cohort of 50-year-old men with GERD symptoms, showed that Cytosponge screening followed by endotherapy reduces EAC mortality and is cost effective [16]. Accurate, minimal invasive and cost-effective screening tools may soon be available for clinicians. Up to now, the effectiveness of endoscopic screening is debatable and there are variable recommendations on it amongst different Medical Societies.

\section{Definition of BE: do we require goblet cells?}

In BE, as a consequence of GERD, the squamous epithelium that normally lines the distal esophagus is replaced by a metaplastic columnar epithelium. Endoscopically this is characterized by the typical salmon color and coarse texture. Histologically it is characterized by specialized intestinal metaplasia with goblet cells. It is a subject of controversy whether or not goblet cells are required as diagnostic criterion for BE. On the one hand, missing goblet cells in a biopsy specimen may represent a sampling error. On the other hand, there is evidence that esophageal cardiac epithelium, although lacking goblet cells, may also predispose to malignancy $[17,18]$. Two retrospective studies evaluated the risk of neoplasia in patients with columnar metaplasia of the esophagus either with or without goblet cells and found non-goblet cell columnar metaplasia to have the same malignant potential $[19,20]$. But the magnitude of this risk is unknown and so is the benefit of endoscopic surveillance. The British Society of Gastroenterology considers esophageal cardiac epithelium as a form of BE. The British guidelines point out that the distinction between columnar-lined esophagus and intestinal metaplasia at the gastric cardia can only be made definitively histologically when columnar mucosa is seen juxtaposed with native anatomical esophageal structures such as submucosal glands and/or gland ducts. But native structures are seen in only $10-15 \%$ of biopsy samples, which implies that in the great majority it is not possible to distinguish between intestinal metaplasia of the cardia and the esophagus. Biopsies of the normal cardia are not recommended routinely but if there is concern about the appearance at the site and after ablation therapy. The presence of intestinal metaplasia is considered as highly corroborative but not specific for a diagnosis of $\mathrm{BE}$, as cardiac intestinal metaplasia cannot be ruled out. However, the guidelines recommend that this information should be recorded and that the diagnosis of $\mathrm{BE}$ should take into account the degree of confidence based on a combined analysis of endoscopic and histopathological criteria [21]. Other societies, including the AGA and the German Society of Gastroenterology, require esophageal biopsies showing intestinal metaplasia with goblet cells to establish the diagnosis [8,22]. After all, intestinal metaplasia is the only type of esophageal columnar epithelium that clearly predisposes to malignancy $[8,22]$.

\section{Diagnosis: can we drop the Seattle protocol with advanced endoscopic imaging?}

To evaluate patients with BE high resolution endoscopy is recommended in order to detect subtle abnormalities of early neoplasia [23]. Endoscopic evidence of BE should be recorded using the Prague criteria [circumferential (C) and maximum (M)] extent of endoscopically visible columnarlined esophagus in centimeters and any separate island above the main columnar-lined segment [24,25]). Current practice standards require the collection of targeted biopsies of every suspicious lesion followed by 4-quadrant biopsies specimens every 1 to $2 \mathrm{~cm}$ of BE (Seattle protocol). This approach is laborintensive, so there has been a great deal of research in imageenhanced technologies.

Chromoendoscopy with contrast enhancing agents such as indigo carmine or acetic acid, virtual chromoendoscopy [Narrow band imaging (NBI, Olympus), Fuji Intelligent Chromo Endoscopy (FICE), and I-scan, Pentax] and confocal laser endomicroscopy, in addition to high-definition standard endoscopy, might increase the diagnostic yield for the detection of dysplastic lesions.

Acetic acid showed a sensitivity of $96 \%$ for the diagnosis of high-grade intraepithelial neoplasia or cancer and a 15-fold increase in neoplasia detection compared to the standardized random biopsy protocol [26,27]. NBI, which highlights surface patterns and vessels, was found to have a sensitivity and specificity of $96 \%$ and $94 \%$ for the diagnosis of HGD in a meta-analysis [28]. In a recent trial, NBI-targeted biopsies showed the same detection rate as high-definition white light examination with the Seattle protocol while requiring fewer biopsies [29]. The Barrett's international NBI Group (BING) developed and validated a NBI classification system to identify dysplasia and EAC in BE. Based on the simple classification of mucosal and vascular patterns as regular (non-dysplastic) and irregular (dysplastic) the BING Criteria could classify BE with $>90 \%$ accuracy and a high level of inter-observer agreement [30].

Overall, advanced imaging techniques increased the diagnostic yield for detection of dysplasia or cancer by $34 \%$ in a recent meta-analysis [31]. In fact they may be very helpful to detect and delineate lesions but their diagnostic power is dependent on the expertise of the individual endoscopist. However, they have not been found to be superior to the standard 4-quadrant random biopsy protocol. Hence, current evidence seems insufficient to change practice. Careful examination using high-resolution endoscopes combined with targeted and 4-quadrant biopsies remains the gold standard [23,24].

\section{Management of BE}

Cancer in $\mathrm{BE}$ is thought to evolve through dysplasia. Dysplasia may be an imperfect marker to predict malignant progression as it can be patchy and therefore missed during routine biopsy sampling. Also, there may be significant interobserver disagreement about its grading [6]. However, dysplasia remains the basis for clinical decision making. 
Endoscopic therapy aims to treat dysplastic precursor tissue to reduce cancer risk. In expert hands, endoscopic therapy of BE-related dysplasia and early neoplasia has shown to be effective and safe. In inexperienced hands, it may be associated with significant complications [32]. Therefore, endoscopic treatment should only be performed in centers with expertise [23]. Before treatment, a lesion should be assessed by an experienced endoscopist, using at least a highresolution endoscope and one of the advanced endoscopic imaging modalities (NBI and/or chromoendoscopy) to determine whether the lesion is suitable for endoscopic treatment, to choose the appropriate resection technique [endoscopic mucosal resection (EMR) or endoscopic submucosal dissection (ESD)], delineate precise margins and to detect other possible lesions [33].

The management of neoplastic BE has changed considerably over the past decade. Today it consists of a multimodal approach combining tissue-acquiring and ablative techniques. Tissue acquiring techniques, which provide tissue specimens for histological examination, include EMR and ESD. Ablative techniques include radiofrequency ablation (RFA), argon plasma coagulation (APC), cryotherapy and photodynamic therapy (PDT). The common goal of ER, ablation or both is to completely eradicate all of the Barrett's metaplasia, dysplastic and non-dysplastic. This concept has shown high rates of disease reversal. A brief algorithm for the endoscopic management of $\mathrm{BE}$ is provided in Fig. 1; details are discussed below.

\section{High-grade dysplasia (HGD) and early cancer}

In BE with HGD or intramucosal cancer endoscopic therapy is well established [34]. In contrast to the low risk of malignant progression in patients with non-dysplastic BE, the risk with HGD is considered high enough to warrant intervention. In a meta-analysis, the annual rate of cancer progression with HGD was calculated approximately $6 \%$ per year, but in endoscopic intervention studies the risk was found to be considerably higher [35,36]. Also, in HGD a risk of occult adenocarcinoma has been reported as high as $40 \%$ [37]. The risk to harbor carcinoma is particularly high in high-grade intraepithelial neoplasia areas that are endoscopically visible. ER of a visible lesion is essential for proper diagnosis and staging. EMR of visible lumps diagnosed with HGD on previous biopsy led in $25-40 \%$ of cases to a histological upgrading to cancer $[38,39]$. Hence, visible lesions should undergo ER. After ER all residual Barrett's mucosa should be eradicated. This two-step concept can significantly reduce the risk of metachronous neoplasia. It remains the question what would be the best ablation technique. Well-studied alternatives are thermal ablation techniques as RFA and APC, PDT, and cryotherapy. Widespread EMR

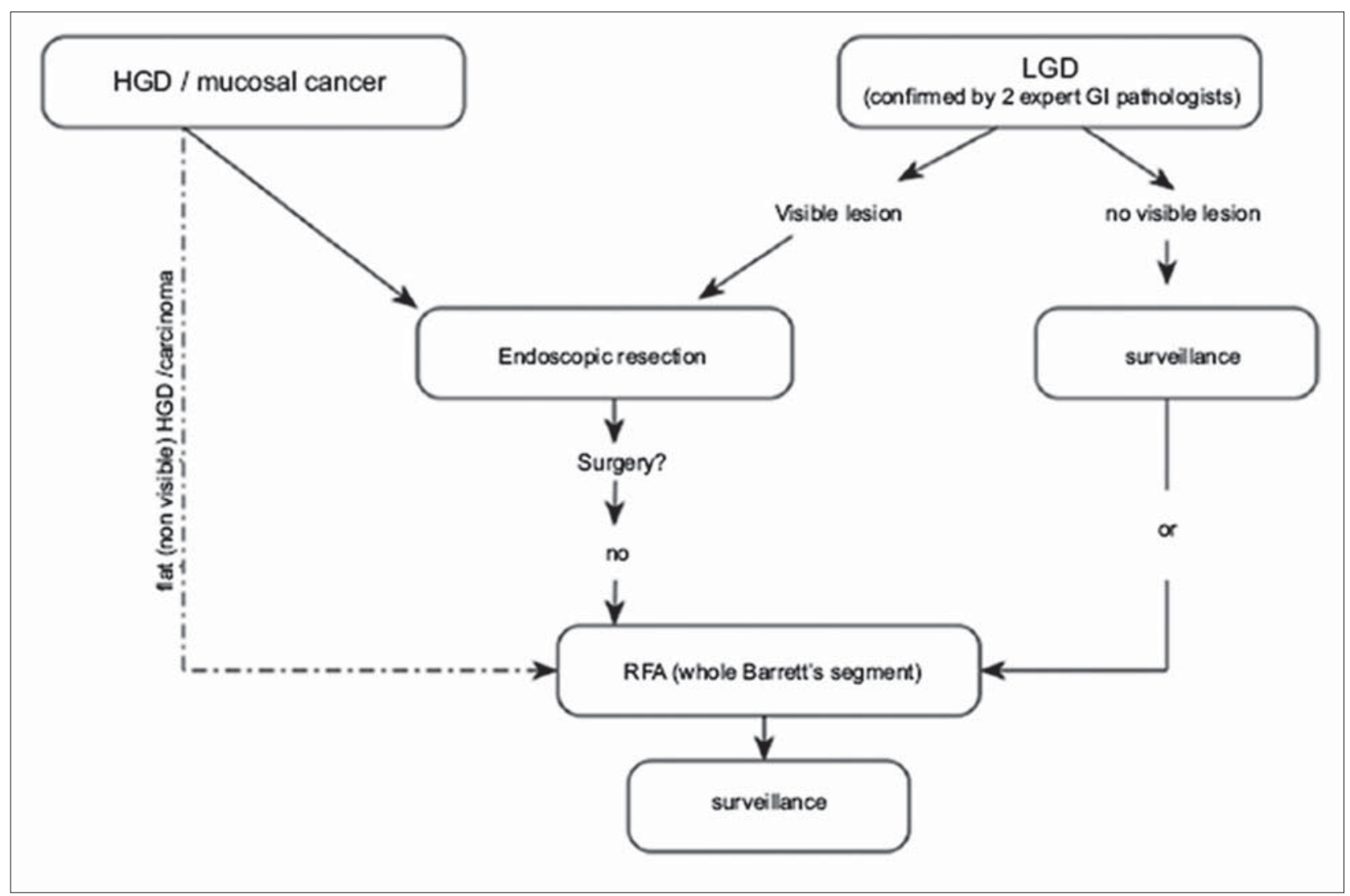

Figure 1 Management of Barrett's esophagus

$H G D$, high-grade dysplasia; LGD, low-grade dysplasia; RFA, radiofrequency ablation 
can cause strictures, especially when more than two thirds of the circumference is removed. PDT plays no significant role any more due to its side effect of photosensitivity of the skin. Although there are no head to head comparative trials RFA seems to be superior and has become the preferred procedure for endoscopic ablation [40,41]. In a meta-analysis including more than 3800 patients RFA achieved complete eradication of dysplasia in $91 \%$ and complete eradication of intestinal metaplasia in $78 \%$ of patients. The most common adverse event was esophageal stricture, which was reported in $5 \%$ of patients [42].

Today the recommended standard of care in patients with HGD or intramucosal cancer is ER of visible lesions followed by RFA of residual Barrett's mucosa [22-43]. This combined approach has shown high rates of disease reversal [44]. However, recurrences have been reported after successful endoscopic therapy. Hence, endoscopic follow up is mandatory. The ESGE recommends regular endoscopic follow up after excision/ablation of BE-associated HGD or mucosal cancer, but more research is necessary to determine the appropriate interval. Empirically, endoscopic follow up is recommended 3-monthly for 1 year and yearly thereafter [33].

- For patients with HGD in an endoscopically visible abnormality, ER is essential for proper diagnosis and staging [23]

- After EMR has removed visible lesions with HGD / $\mathrm{T} 1 \mathrm{~m}$, the remaining BE segment should be eradicated regardless of whether or not it includes the presence or absence of dysplasia [23]

- RFA is currently the best available ablation technique for treatment of flat HGD and for eradication of residual BE mucosa after focal EMR [23]

If adenocarcinoma is found in the EMR specimen the risk of lymph node metastasis has been shown to correlate with the depth of invasion. In patients with mucosal neoplasm lymph node metastases are present in less than $2 \%$ but in patients with tumor infiltration into the deep submucosa in more than $20 \%$ [45]. In contrast to surgery, endoscopic therapy does not have the potential to cure neoplasm that has metastasized to regional lymph nodes. Therefore, ER is considered curative for intramucosal carcinomas that are well or moderately differentiated (G1-G2) without lymphatic or vascular invasion (L0, V0) [33]. Endoscopic en bloc R0 resection of a sm1 $(<500 \mu \mathrm{m})$ low risk tumor (G1-2, L0 and V0, size $<3 \mathrm{~cm})$ is considered potentially curative [33]. Manner et al reported for these lesions a low risk of lymph node metastasis (1.4\%), but only few patients were included in that study [46]. So the risk of lymph node metastasis should be balanced against the risk of surgery for the individual patient in a multidisciplinary discussion [33].

Surgery is recommended in the presence of [33]:

- Lymph vascular invasion (L1,V1)

- Deeper infiltration of the submucosa than $\operatorname{sml}(\geq 500 \mu \mathrm{m})$

- Poorly differentiated tumor

- Positive vertical margins (R1 vertical)
If the horizontal margin is positive ( $\mathrm{R} 1$ horizontal) or the tumor was resected piece meal and no other high-risk criteria are met, close endoscopic surveillance/treatment is recommended rather than surgery [33].

The standard for ER of Barrett's neoplasia in current clinical practice is EMR. But in lesions $>15 \mathrm{~mm}$ EMR entails piece meal resection, associated with higher recurrence rates and hampers histopathological assessment of free margins [47]. ESD allows the en bloc resection of a lesion regardless of its size (Fig.2). With en bloc resection the histopathological evaluation is improved and an adequate assessment of R0-status and curative resection as defined to oncological standards is possible. The efficiency and safety of ESD with high success rates have been demonstrated by Asian studies [48-50]. But in Asian countries $\mathrm{BE}$ and adenocarcinoma are still rare. Therefore, available data on ESD in BE are scarce. Nevertheless, it has been shown that also in western countries ESD of Barrett's neoplasia is feasible with en bloc resection rates $>95 \%$, R0-resection rates $>80 \%$, and complication rates comparable to EMR [51-53].

In the presence of HGD or intramucosal cancer without visible lesions (flat $\mathrm{HGD}$ /intramucosal cancer) RFA is recommended [21-23,33]. Widespread EMR can cause strictures, especially when more than two thirds of the circumference is removed. RFA has been compared with stepwise ER for complete eradication of BE containing HGD/ mucosal cancer. Both methods showed equivalent efficacy but radical ER was associated with higher stricture rates and therapeutic sessions [54]. If HGD or intramucosal cancer are confirmed and there are no visible lesions after expert high resolution endoscopy review, then ablative therapy is the treatment of choice [21]. But to reemphasize it: RFA should only be used as a primary treatment modality in the case of flat HGD/intramucosal cancer. All visible lesions should be resected to provide adequate histological assessment, even if this demands circumferential EMR or ESD. Resulting strictures do respond well to dilatation and there exist strategies for stricture prevention, such as steroid administration.

\section{LGD}

The management of LGD is confounded by uncertainty of its natural history and difficulties in making the diagnosis. The diagnosis of LGD in BE is a subject of high interobserver variability among pathologists and can be challenging in the presence of inflammation. As demonstrated in a recent Dutch

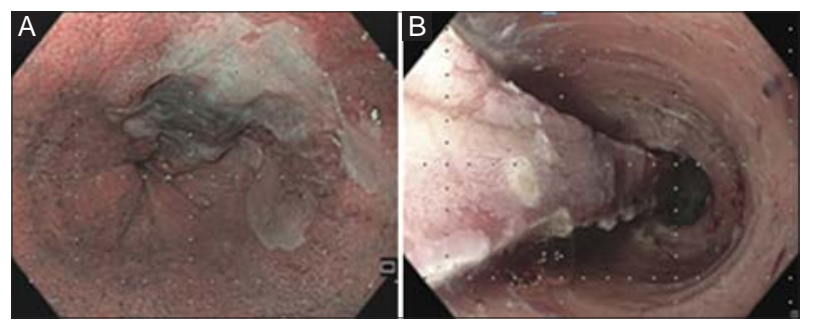

Figure 2 (A) Early esophageal adenocarcinoma (narrow band imaging); (B) after endoscopic submucosal dissection 
study, LGD in BE seems to be an over diagnosed and yet underestimated entity [6]. In this study $85 \%$ of patients who were initially diagnosed with LGD were down staged to either non-dysplastic or to indefinite for dysplasia (IND) after review by two expert GI pathologists. So it seems essential that the diagnosis is confirmed by at least two GI expert pathologists. The trial also showed that for patients with a consensus diagnosis of LGD, the cumulative risk of progression to HGD or carcinoma was alarming $85 \%$ in 109 months and the incidence rate for HGD or carcinoma $13.4 \%$ per patient per year. For down staged patients the corresponding incidence rate was $0.49 \%$. Faced with this data gastroenterology societies propose that the diagnosis of dysplasia in BE should be confirmed by at least one additional pathologist, preferably one who is an expert in esophageal/gastrointestinal (GI) histopathology $[21,22]$. This recommendation takes in account the great medical importance of a "true" diagnosis of LGD but implicates challenges in its practical implementation (definition/qualification of an expert pathologist, independent evaluation, down-staging of diagnoses, financial aspects etc.).

The finding of an endoscopically visible lesion in the setting of biopsy-detected LGD is of special importance as it may contain HGD or invasive cancer. Hence, visible lesions in confirmed LGD should be resected endoscopically to enable accurate histological assessment [55]. ER may result in a change of histological diagnosis, as shown in a multicenter study, where ER in patients diagnosed with LGD on biopsy led to upstaging in $33.3 \%$ and downstaging in $13.3 \%$ [56]. If HGD or mucosal cancer is detected ER should be followed by ablation [55].

- We recommend that in the case of BE visible lesions in diagnosed LGD (or IND), ER should be followed by ablation if $\mathrm{HGD}$ or intramucosal cancer is detected, rather than continued surveillance [55]

Ablation of BE with only LGD remains controversial because there is no clarity on cancer risk. As mentioned above, LGD generally seems to be overcalled but in those patients with LGD confirmed by at least two expert GI pathologists the risk of neoplastic progressions is considerably high. There are several studies that also indicate some clinical risk features such as multifocality of LGD and the length of BE-segment [57]. RFA can significantly reduce the risk of neoplastic progression to HGD/EAC. In the "SURF" trial it decreased the progression rate from $26.5 \%$ (control) to $1.5 \%$ (RFA) [58].

\section{Non-dysplastic BE}

Noting the success of RFA in eradicating Barrett's metaplasia some physicians have proposed that RFA should be offered to all BE patients rather than to restrict it to patients with dysplasia. But patients with no dysplastic BE have a very low risk to develop HGD or EAC. Recent studies show that the risk may be as low as $0.12-0.33 \%$ per year [3]. This low risk does not weigh out potential therapy associated risks and does not justify therapeutic intervention. Visible lesions in non-dysplastic BE (as well as visible lesions in BE with LGD or IND) should undergo ER to enable accurate histological assessment [55].

- We suggest against using ER in patients with nondysplastic BE and no visible lesion (harms outweigh benefits) [55]

\section{Endoscopic surveillance}

Endoscopic surveillance for $\mathrm{BE}$ is recommended by all Medical Societies. It is based on the assumption that the transition from BE to EAC progresses through LGD and HGD, thus justifying endoscopic surveillance for these premalignant stages. To date, the only evidence supporting this practice comes from observational studies reporting that patients whose Barrett's carcinoma was diagnosed during surveillance endoscopy have earlier stage tumor and better survival. The recommendations vary amongst different Medical Societies and further data on optimal intervals and protocols for biopsy collection is needed.

Broadly speaking, in non-dysplastic BE surveillance endoscopy is recommended every 3-5 years. In the presence of LGD, confirmed by at least two expert GI pathologists, without visible lesions surveillance endoscopy every 6-12 months or eradication therapy is recommended [7].

Since recurrences of Barrett's metaplasia after apparently successful eradication are possible and recurrence rates up to $33 \%$ have been reported [59], patients should continue to undergo endoscopic surveillance even after therapy. Empirically, in patients treated for HGD, endoscopic follow up is recommended 3-monthly for 1 year and yearly thereafter.

\section{Practical impact}

- BE is a combined endoscopic and pathological diagnosis

- The Seattle protocol (4-quadrant biopsies every 1 to $2 \mathrm{~cm}$ of $\mathrm{BE}$ and of every suspicious lesion) remains the standard; advanced imaging techniques may increase the diagnostic yield

- For any degree of dysplasia, at least two expert GI pathologists are required to confirm the diagnosis

- Visible lesions should be endoscopically resected to enable accurate histological assessment

- In HGD/mucosal cancer ER of visible lesions followed by field ablation of the whole Barrett's segment with RFA is now the standard of care

- In LGD (confirmed by at least two expert GI pathologists) with visible lesions ER should be performed. Without visible lesions surveillance endoscopy every 6-12 months or eradication therapy is recommended

- In non-dysplastic BE the risk of progression is low. Surveillance endoscopies are recommended every 3-5 years

- Recurrences after apparently successful eradication of 
Barrett's metaplasia are possible. Further endoscopic surveillance is indispensable.

\section{References}

1. Hur C, Miller M, Kong CY, et al. Trends in esophageal adenocarcinoma incidence and mortality. Cancer 2013;119:1149-1158.

2. Verbeek RE, Leenders M, Ten Kate FJ, et al. Surveillance of Barrett's esophagus and mortality from esophageal adenocarcinoma: a population-based cohort study. Am $J$ Gastroenterol 2014; 109:1215-1222.

3. Hvid-Jensen F, Pedersen L, Drewes AM, Sørensen HT, FunchJensen P. Incidence of adenocarcinoma among patients with Barrett's esophagus. N Engl J Med 2011;365:1375-1383.

4. Desai TK, Krishnan K, Samala N, et al. The incidence of oesophageal adenocarcinoma in non-dysplastic Barrett's oesophagus: a metaanalysis. Gut 2012;61:970-976.

5. Bhat S, Coleman HG, Yousef F, et al. Risk of malignant progression in Barrett's esophagus patients: results from a large populationbased study. JNCI J Natl Cancer Inst 2011;103:1049-1057.

6. Curvers WL, ten Kate FJ, Krishnadath KK, et al. Low-grade dysplasia in Barrett's esophagus: overdiagnosed and underestimated. Am J Gastroenterol 2010;105:1523-1530.

7. Spechler SJ, Souza RF. Barrett's esophagus. N Engl J Med 2014;371:836-845

8. Spechler SJ, Sharma P, Souza RF, et al. American Gastroenterological Association medical position statement on the management of Barrett's esophagus. Gastroenterology 2011;140:1084-1091.

9. Rex DK, Cummings OW, Shaw M, et al. Screening for Barrett's esophagus in colonoscopy patients with and without heartburn. Gastroenterology 2003;125:1670-1677.

10. Chak A, Faulx A, Eng C, et al. Gastroesophageal reflux symptoms in patients with adenocarcinoma of the esophagus or cardia. Cancer 2006;107:2160-2166.

11. Gaddam S, Singh M, Balasubramanian G, et al. Persistence of nondysplastic Barrett's esophagus identifies patients at lower risk for esophageal adenocarcinoma: Results from a large multicenter cohort. Gastroenterology 2013;145:548-553.

12. Di Pietro M, Alzoubaidi D, Fitzgerald RC. Barrett's esophagus and cancer risk: how research advances can impact clinical practice. Gut Liver 2014;8:356-370.

13. Gregson EM, Fitzgerald RC. Biomarkers for dysplastic Barrett's: ready for prime time? World J Surg 2015;39:568-577.

14. Di Pietro M, Chan D, Fitzgerald R, Wang K. Screening for Barrett's esophagus. Gastroenterology 2015;148:912-923.

15. Kadri SR, Lao-Sirieix P, Donovan M, et al. Acceptability and accuracy of a non-endoscopic screening test for Barrett's oesophagus in primary care: cohort study. BMJ 2010;341:c4372.

16. Benaglia T, Sharples LD, Fitzgerald RC, Lyratzopoulos G. Health benefits and cost effectiveness of endoscopic and nonendoscopic cytosponge screening for Barrett's esophagus. Gastroenterology 2013;144:62-73.

17. Takubo K, Aida J, Naomoto Y, et al. Cardiac rather than intestinaltype background in endoscopic resection specimens of minute Barrett adenocarcinoma. Hum Pathol 2009;40:65-74.

18. Liu W, Hahn H, Odze RD, Goyal RK. Metaplastic esophageal columnar epithelium without goblet cells shows DNA content abnormalities similar to goblet cell containing epithelium. Am J Gastroenterol 2009;104:816-824.

19. Gatenby PAC, Ramus JR, Caygill CPJ, Shepherd NA, Watson A. Relevance of the detection of intestinal metaplasia in non-dysplastic columnar-lined oesophagus. Scand J Gastroenterol
2008;43:524-530.

20. Kelty CJ, Gough MD, Van Wyk Q, Stephenson TJ, Ackroyd R. Barrett's oesophagus: intestinal metaplasia is not essential for cancer risk. Scand J Gastroenterol 2007;42:1271-1274.

21. Fitzgerald RC, di Pietro M, Ragunath K, et al. British Society of Gastroenterology guidelines on the diagnosis and management of Barrett's oesophagus. Gut 2014;63:7-42.

22. Koop H, Fuchs KH, Labenz J, et al. S2k Guideline: Gastroesophageal reflux disease guided by the German Society of Gastroenterology. Z Gastroenterol 2014;52:1299-1346.

23. Bennett C, Vakil N, Bergman J, et al. Consensus statements for management of Barrett's dysplasia and early-stage esophageal adenocarcinoma, based on a Delphi process. Gastroenterology 2012;143:336-346.

24. Sharma P, Katzka DA, Gupta N. Quality indicators for the management of Barrett's esophagus, dysplasia, and esophageal adenocarcinoma: international consensus recommendations from the American Gastroenterological Association Symposium. Gastroenterology 2015;149:1599-1606.

25. Sharma P, Dent J, Armstrong D, et al. The development and validation of an endoscopic grading system for Barrett's esophagus: the Prague C \& M criteria. Gastroenterology 2006;131:1392-1399.

26. Longcroft-Wheaton G, Duku M, Mead R, Poller D, Bhandari P. Acetic acid spray is an effective tool for the endoscopic detection of neoplasia in patients with Barrett's esophagus. Clin Gastroenterol Hepatol 2010;8:843-847.

27. Tholoor S, Bhattacharyya R, Tsagkournis O, Longcroft-Wheaton G, Bhandari P. Acetic acid chromoendoscopy in Barrett's esophagus surveillance is superior to the standardized random biopsy protocol: results from a large cohort study (with video). Gastrointest Endosc 2014;80:417-424.

28. Mannath J, Subramanian V, Hawkey CJ, Ragunath K. Narrow band imaging for characterization of high grade dysplasia and specialized intestinal metaplasia in Barrett's esophagus: a metaanalysis. Endoscopy 2010;42:351-359.

29. Sharma P, Hawes RH, Bansal A, et al. Standard endoscopy with random biopsies versus narrow band imaging targeted biopsies in Barrett's oesophagus: a prospective, international, randomised controlled trial. Gut 2013;62:15-21.

30. Sharma P, Bergman J, Goda K, et al. Development and validation of a classification system to identify gigh-grade dysplasia and esophageal adenocarcinoma in Barrett's esophagus using narrowband imaging. Gastroenterology 2016;150:591-598.

31. Qumseya BJ, Wang $\mathrm{H}$, Badie $\mathrm{N}$, et al. Advanced imaging technologies increase detection of dysplasia and neoplasia in patients with Barrett's esophagus: a meta-analysis and systematic review. Clin Gastroenterol Hepatol 2013;11:1562-1570.

32. Van Vilsteren FGI, Pouw RE, Herrero LA, et al. Learning to perform endoscopic resection of esophageal neoplasia is associated with significant complications even within a structured training program. Endoscopy 2012;44:4-12.

33. Pimentel-Nunes, et al. Endoscopic submucosa dissection: European Society of Gastrointestinal Endoscopy (ESGE) Guideline. Endoscopy 2015;47:829-854.

34. Pech O, May A, Manner H, et al. Long-term efficacy and safety of endoscopic resection for patients with mucosal adenocarcinoma of the esophagus. Gastroenterology 2014;146:652-660.

35. Rastogi A, Puli S, El-Serag HB, Bansal A, Wani S, Sharma P. Incidence of esophageal adenocarcinoma in patients with Barrett's esophagus and high-grade dysplasia: a meta-analysis. Gastrointest Endosc 2008;67:394-398.

36. Shaheen NJ, Sharma P, Overholt BF, et al. Radiofrequency ablation in Barrett's esophagus with dysplasia. $N$ Engl $J$ Med 2009;360:2277-2288.

37. Wright TA. High-grade dysplasia in Barrett's oesophagus. Br J Surg 1997;84:760-766. 
38. Peters FP, Brakenhoff KP, Curvers WL, et al. Histologic evaluation of resection specimens obtained at 293 endoscopic resections in Barrett's esophagus. Gastrointest Endosc 2008;67:604-609.

39. Pech O, Gossner L, Manner H. Prospective evaluation of the macroscopic types and location of early Barrett's neoplasia in 380 lesions. Endoscopy 2007;39:588-593.

40. Blevins CH, Iyer PG. Endoscopic therapy for Barrett's oesophagus. Best Pract Res Clin Gastroenterol 2015;29:167-177.

41. Haidry R, Lovat L, Sharma P. Radiofrequency ablation for Barrett's dysplasia: past, present and the future? Curr Gastroenterol Rep 2015;17:13.

42. Orman ES, Li N, Shaheen NJ. Efficacy and durability of radiofrequency ablation for Barrett's esophagus: systematic review and meta-analysis. Clin Gastroenterol Hepatol 2013;11:1245-1255.

43. Porschen R, Buck A, Fischbach W, et al. S3-Leitlinie diagnostik und therapie der plattenepithelkarzinome und adenokarzinome des Ösophagus. Z Gastroenterol 2015;53:1288-1347 (S3-Guideline on diagnosis and therapy of esophageal cancer, German Society of Gastroenterology).

44. Phoa KN, Pouw RE, Bisschops R, et al. Multimodality endoscopic eradication for neoplastic Barrett oesophagus: results of an European multicentre study (EURO-II). Gut 2016;65:555-562.

45. Dunbar KB, Spechler SJ. The risk of lymph-node metastases in patients with high-grade dysplasia or intramucosal carcinoma in Barrett's esophagus: a systematic review. Am J Gastroenterol 2012;107:850-862.

46. Manner H, May A, Pech O, et al. Early Barrett's carcinoma with "low risk" submucosal invasion: long term results of endoscopic resection with a curative intent. Am J Gastroenterol 2008;103:2589-2597.

47. Pech O, Behrens A, May A. Long-term results and risk factor analysis for recurrence after curative endoscopic therapy in 349 patients with high-grade intraepithelial neoplasia and mucosal adenocarcinoma in Barrett's oesophagus. Gut 2008;57:1200-1206.

48. Kakushima N, Yahagi N, Fujishiro M. Efficacy and safety of endoscopic submucosal dissection for tumors of the esophagogastric junction. Endoscopy 2006;38:170-174.

49. Hoteya S, Matsui A, Iizuka T. Comparison of the clinicopathological characteristics and results of endoscopic submucosal dissection for esophagogastric junction and non-junctional cancers. Digestion 2013;87:29-33.

50. Hirasawa K, Kokawa A, Oka H. Superficial adenocarcinoma of the esophagogastric junction: long-term results of endoscopic submucosal dissection. Gastrointest Endosc 2010;72:960-966.

51. Probst A, Aust D, Märkl B, Anthuber M, Messmann H. Early esophageal cancer in Europe: endoscopic treatment by endoscopic submucosal dissection. Endoscopy 2015;47:113-121.

52. Chevaux JB, Piessevaux H, Jouret-Mourin A, et al. Clinical outcome in patients treated with endoscopic submucosal dissection for superficial Barrett's neoplasia. Endoscopy 2015;47:103-112.

53. Höbel S, Dautel P, Baumbach R, et al. Single center experience of endoscopic submucosal dissection (ESD) in early Barrett's adenocarcinoma. Surg Endosc 2015;29:1591-1597.

54. van Vilsteren FG, Pouw RE, Seewald S, et al. Stepwise radical endoscopic resection versus radiofrequency ablation for Barrett's oesophagus with high-grade dysplasia or early cancer: a multicentre randomized trial. Gut 2011;60:765-773.

55. Bennett C, Moayyedi P, Corley DA, et al. BOB CAT: A largescale review and Delphi Consensus for management of Barrett's esophagus with no dysplasia, indefinite for, or low-grade dysplasia. Am J Gastroenterol 2015;110:662-682.

56. Wani S, Abrams J, Edmundowicz SA, et al. Endoscopic mucosal resection results in change of histologic diagnosis in Barrett's esophagus patients with visible and flat neoplasia: a multicenter cohort study. Dig Dis Sci 2013;58:1703-1709.

57. Thota PN, Lee HJ, Goldblum JR, et al. Risk stratification of patients with barrett's esophagus and low-grade dysplasia or indefinite for dysplasia. Clin Gastroenterol Hepatol 2015;13:459-465.

58. Phoa KN, van Vilsteren FG, Weusten BL, et al. Radiofrequency ablation vs endoscopic surveillance for patients with Barrett esophagus and low-grade dysplasia: a randomized clinical trial. JAMA 2014;311:1209-1217.

59. Gupta M, Iyer PG, Lutzke L, et al. Recurrence of esophageal intestinal metaplasia after endoscopic mucosal resection and radiofrequency ablation of Barrett's esophagus: eesults from a US multicenter consortium. Gastroenterology 2013;145:79-86. 